\title{
Charging dynamics of InAs self-assembled quantum dots
}

\author{
G. Medeiros-Ribeiro, ${ }^{*}$ J. M. Garcia, and P. M. Petroff \\ Center for Quantized Electronic Structures (QUEST) and Materials Department, University of California, Santa Barbara, \\ Santa Barbara, California 93106
}

(Received 18 April 1997)

\begin{abstract}
We present results on the dynamics of the charging of arrays of InAs self-assembled quantum dots (SAQD's). An equivalent circuit used for metal-insulator-semiconductor-field-effect transistor structures is applied and successfully predicts the observed behavior of the capacitance and conductance dependence on the excitation frequency. The Coulomb blockade effect also plays a role in the frequency-dependent spectra. A Coulomb blockade in this system was observed in arrays of over $10^{7}$ SAQD's, demonstrating the good size uniformity of such a system. [S0163-1829(97)00932-6]
\end{abstract}

The so called self-assembled systems have been receiving a great amount of attention in the past few years as a mean of mass producing low dimensional systems in a relatively simple way. ${ }^{1}$ Optical and transport properties of such structures have demonstrated the potential use of this scheme on new devices based on three-dimensional (3D) quantum confinement and Coulomb blockade effect for the InAs/GaAs system. The InAs self-assembled quantum dots (SAQD's) produced this way present remarkable uniformity $(10 \%)$. Of the lattice mismatched III-V systems it is the one that most successfully demonstrated 3D quantum confinement effects and Coulomb blockade effects. Laser operation has been demonstrated by several groups ${ }^{2,3}$ and memory schemes ${ }^{4}$ have also been proposed with promising applications of InAs SAQD's in devices.

The quantum confinement properties of this system have been studied by capacitance spectroscopy and far infrared absorption (FIR) where hole and electron states have been assessed $^{5}$ along with the dependence of the excited levels in a magnetic field. ${ }^{6,7}$ Temperature dependent capacitance spectroscopy ${ }^{8}$ has been done in such systems, where quantum confinement properties have been probed. Coulomb blockade in this system has been carefully studied using capacitance spectroscopy ${ }^{9}$ and a theoretical treatment has been developed to predict effects of back contact proximity, dotdot interaction, and Coulomb disorder in the charging process of large ensembles of InAs SAQD's. The study of a small number of islands has revealed also fine structure, presumably from the interaction of neighboring SAQD's, behaving as molecules. ${ }^{10}$ Finally, the assessment of transport characteristics of a single SAQD has been done with ballistic-electron-emission microscopy (BEEM). ${ }^{11}$

The objective of this paper is to investigate the frequency dependence of the admittance $Y(\omega)=G(\omega)+\omega C(\omega)$ of an ensemble of InAs SAQD's and the effects of the ac excitation voltage on the Coulomb blockade and on the charging dynamics in these structures. These results should give a few guidelines in the design of device structures where charge transport from and to SAQD's is involved.

The samples used in this study were the same used in Ref. 9. There, InAs SAQD's were embedded in between an undoped GaAs tunneling barrier and a GaAs/AlAs undoped supperlattice. Back contacts were made to an $n+$ layer be- neath the tunneling barrier of thickness $t_{b}$, and the Schottky top contact a distance $t_{\text {tot }}$ above the back contact, giving a lever arm ratio $t_{\text {tot }} / t_{b}$. Therefore, all voltages differences can be converted into energy differences by dividing them by $t_{\text {tot }} / t_{b}$. For the frequency-dependence study, we chose the sample with $t_{b}=450 \AA$ and $t_{\text {tot }} / t_{b}$ of 6 . The capacitance experiments were performed on samples immersed in liquid He. A dual-phase lock-in amplifier was used to measure the admittance characteristics, and both capacitance and conductance were recorded as a function of the applied bias. The Schottky diodes formed with the samples were defined by conventional lithography to disks of diameter $340 \mu \mathrm{m}$. The measurements were therefore performed in ensembles of approximately $1 \times 10^{7}$ SAQD's, since typical densities consisted of $1 \times 10^{10} \mathrm{~cm}^{-2}$.

It can be shown that the density of states of a given quantum system can be extracted from capacitance experiments. ${ }^{5,12}$ Therefore, the SAQD density can be extracted if one knows the degeneracy factor for each level. Using symmetry arguments and results from the capacitance and far infrared absorption (FIR) experiments ${ }^{7}$ one gets that the first state is occupied by two electrons, the second by four and so on, and for each electron added to the SAQD system one must add the Coulomb charging energy. For systems where the spread in size of SAQD's is small, the charging energy of a single electron can be observed ${ }^{7}$ in large ensembles of SAQD's. Nevertheless, to extract exactly the density of SAQD's, one must use an equivalent circuit to describe the transport experiments. For similar sample structures, it has been shown ${ }^{12-15}$ that one can describe tunneling capacitor structures by a series combination of a capacitor $C_{\text {low }}$ representing the top insulating layer comprising the superlattice and a parallel combination of a resistor $R_{\text {tunn }}$ and a capacitor $C_{\text {tunn }}$ describing the tunneling barrier. Figure 1 shows the equivalent circuit and the corresponding band diagram for the structure studied in this work. Therefore, in order to extract these elements separately one can perform frequency-dependent experiments, where at the high frequency limit the series combination of $C_{\text {low }}$ and $C_{\text {tunn }}$ will be measured and at low frequencies $C_{\text {low }}$ will be measured. The SAQD density of states can then be extracted from this type of measurement. We have a Debye type relaxation 


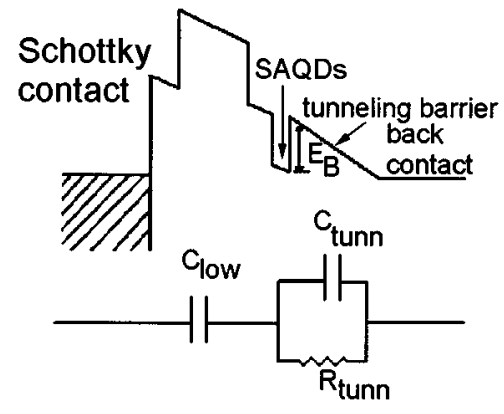

FIG. 1. Sample band diagram and proposed equivalent circuit.

mechanism for the charging process of the SAQD's and this can de described by the following set of equations: ${ }^{12,13,16}$

$$
\begin{gathered}
C(\omega)=\frac{C_{\text {high }} C_{\text {low }}\left[1+\left(\omega / \omega_{\text {peak }}\right)^{2}\right]}{C_{\text {high }}+C_{\text {low }}\left(\omega / \omega_{\text {peak }}\right)^{2}}, \\
\frac{G(\omega)}{\omega}=2 \sqrt{C_{\text {high }} C_{\text {low }}} \frac{\left(C_{\text {low }}-C_{\text {high }}\right) \omega / \omega_{\text {peak }}}{C_{\text {high }}+C_{\text {low }}\left(\omega / \omega_{\text {peak }}\right)^{2}},
\end{gathered}
$$

where $C_{\text {high }}$ is a series combination of $C_{\text {low }}$ and $C_{\text {tunn }}$ and $\omega_{\text {peak }}$ is the angular frequency $1 / R_{\text {tunn }} C_{\text {tunn }}$. Figure 2 shows the experimentally measured frequency $f=\omega / 2 \pi$ dependence of the capacitance $C(V)$ and normalized conductance $G(V) / \omega$ characteristics. The arrows indicate the direction of increasing frequencies. In the $C(V)$ characteristics at low

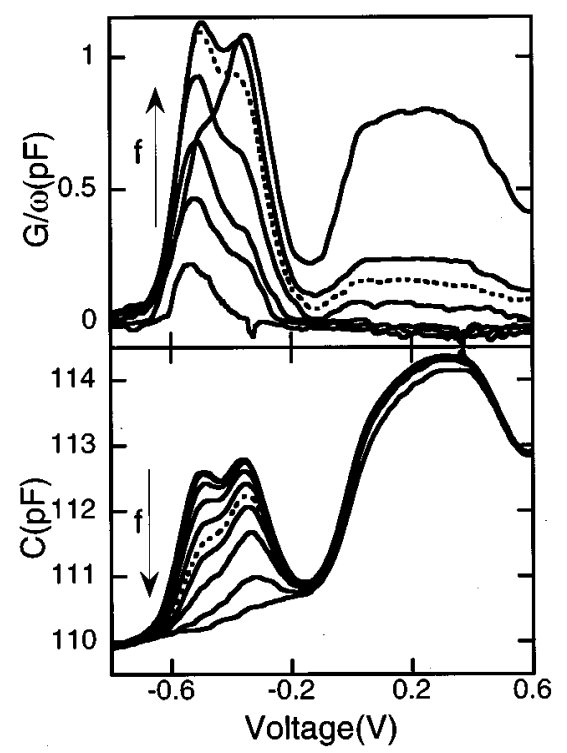

FIG. 2. Frequency dependence of $G(V) / \omega$ (top) and $C(V)$ (bottom) characteristics. The arrow indicates the direction of increasing frequencies. The frequencies used for the $G(V) / \omega$ traces were 100 $\mathrm{Hz}, 200 \mathrm{~Hz}, 325 \mathrm{~Hz}, 500 \mathrm{~Hz}, 1 \mathrm{kHz}, 1.2 \mathrm{kHz}$, and $4 \mathrm{kHz}$; as for the $C(V)$ traces in addition to the aforementioned frequencies we used $730 \mathrm{~Hz}, 2 \mathrm{kHz}$, and $8.2 \mathrm{kHz}$. The dotted $C(V)$ and $G(V) / \omega$ were measured at $\omega_{\text {peak }}(\mathrm{f}=1 \mathrm{kHz})$ for the ground state of the SAQD ensemble, which occurred at $-0.5 \mathrm{~V}$. At this frequency, half of the electrons tunnel into the SAQD's within one cycle of the ac excitation frequency (see text).

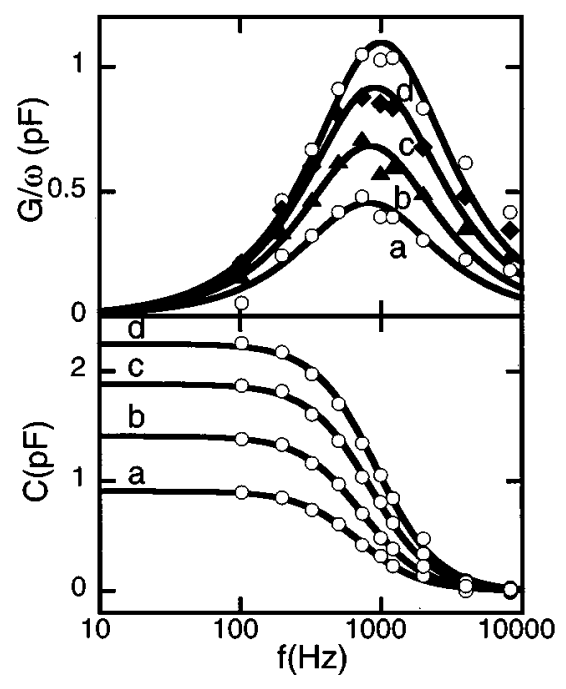

FIG. 3. Normalized conductance (top) and capacitance (bottom) at voltages $a=-0.6 \mathrm{~V}, b=-0.575 \mathrm{~V}, c=-0.55 \mathrm{~V}$, and $d=-0.525 \mathrm{~V}$, together with the corresponding fits (solid lines) according to Eq. (1) (see text). The symbols correspond to experimental data.

frequencies the signature of the first two single electron loading events into the ground level of the InAs SAQD ensemble can be readily observed. The amplitudes of the capacitance peaks decrease with increasing frequency, and at $\omega=\omega_{\text {peak }}$ we have exactly half of the electrons tunneling into the SAQD ensemble within one period of the excitation frequency. At higher frequencies we do not have a tunneling event within one cycle of the ac signal. Electron levels near and below the quasi Fermi level established by the applied dc bias remain occupied during an ac voltage cycle, and the tunneling events occur exclusively due to the dc bias. It can be seen that for each dot size, now characterized by a tunneling event at a given voltage $V$, there is one $f_{\text {peak }}$, and the deeper the level is, the higher the tunneling resistance is and therefore the lower $f_{\text {peak }}$. Indeed, within the range of frequencies utilized in this experiment, electrons tunneling in the first excited state did seem to have enough time to do so, except maybe for frequencies of tens of $\mathrm{kHz}$. As for the conductance characteristics, the evidences of tunneling out of phase become noticeable at much lower frequencies. The conductance signal changes by one order of magnitude over the frequency and bias ranges utilized in this experiment, whereas capacitance signal changes by no more than $2 \%$. The conductance signal is, therefore, a clear indication of nonequilibrium tunneling events.

In Fig. 3 we selected four different voltages and displayed the capacitance and normalized conductance at these voltages as a function of the measurement frequency, together with the fits to $C(\omega)-C_{\text {high }}$ and $G(\omega) / \omega$ according to Eq. (1). The fit to both capacitance and normalized conductance is very good within the experimental uncertainties and we can therefore assume that the proposed equivalent circuit describes well the system studied. Both capacitance and normalized conductance are proportional to the density of states. ${ }^{13}$ It is important to note also that the linewidth of the normalized conductance peak $G\left(\omega_{\text {peak }}\right) / \omega_{\text {peak }}$ is not related to size nonuniformities of the SAQD ensemble, but to the nature of the charging process in such structures. 


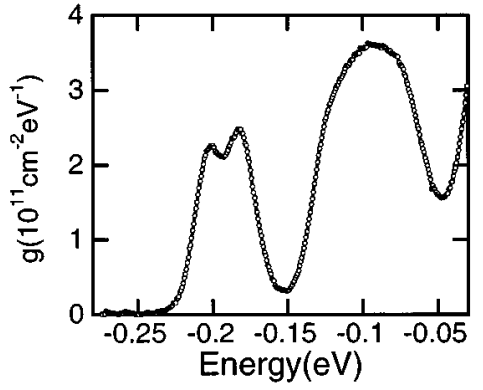

FIG. 4. Calculated density of states from the $C_{\text {low }}(V)$ and $C_{\text {high }}(V)$ characteristics and Eq. (2).

Once the circuit elements can be extracted for each voltage, one can relate the capacitance directly to the density of states per unit area, $g(E)$, of the system by solving Poisson's equation for this system. Assuming that there is no charge in the insulating layers, and that there is no band bending for each electron loaded in the SAQD's array, one gets: ${ }^{12,16}$

$$
g(E)=\frac{C_{\text {tunn }}}{q^{2}}\left(\frac{C_{\text {low }}}{C_{\text {high }}}-1\right)\left[1+C_{\text {low }}\left(\frac{1}{C_{\text {tunn }}}-\frac{1}{C_{\text {high }}}\right)\right]^{-1},
$$

where $C_{\text {tunn }}$ is simply $\varepsilon \varepsilon_{0} / t_{b}\left(\mathrm{pF} / \mathrm{cm}^{2}\right)$, being $\varepsilon$ the GaAs dielectric constant, and $\varepsilon_{0}$ the vacuum permittivity, and $q$ is the elemental charge. Figure 4 represents such a plot, where $C_{\text {low }}$ at higher voltages (loading of first excited state and above) was approximated by a linear relationship extrapolated from negative voltages. The energy axis was calculated using the linear relationship between energy and voltage through the lever arm according to the procedure described elsewhere..$^{5}$ One can see that both linear approximations work reasonably well for the two first electron tunneling events in the ground state of the SAQD ensemble. Nevertheless, this approximation progressively worsens as the number of electron is increased. For the calculation of SAQD's densities one does not need to include all states; by integrating the $g(E)$ over the energy range correspondent to the two first tunneling events one gets $1.2 \times 10^{10} \mathrm{~cm}^{-2}$ for the number of charges. Therefore, the SAQD's density $6 \times 10^{9} \mathrm{~cm}^{-2}$ is in agreement with TEM experiments performed in similar samples.

In Fig. 5 we display the dependence of the tunneling time $\tau=2 \pi / \omega_{\text {peak }}=1 / f_{\text {peak }}$ as a function of the square root of the energy barrier height $E_{B}$. Displayed on the right axis is the dependence of the tunneling resistance $R_{\text {tunn }}$ as a function of $\sqrt{E_{B}}$. One can calculate this quantity using $\tau$ and $C_{\text {tunn }}$, which can be calculated from the sample structure. For this particular sample structure we get $C_{\text {tunn }}$ of $255 \mathrm{nF} / \mathrm{cm}^{2}$, so for our device of area $0.001 \mu \mathrm{m}^{2}$ that is $255 \mathrm{pF}$. The solid lines on both $R_{\text {tunn }}$ and $\tau$ correspond to exponential fits. From

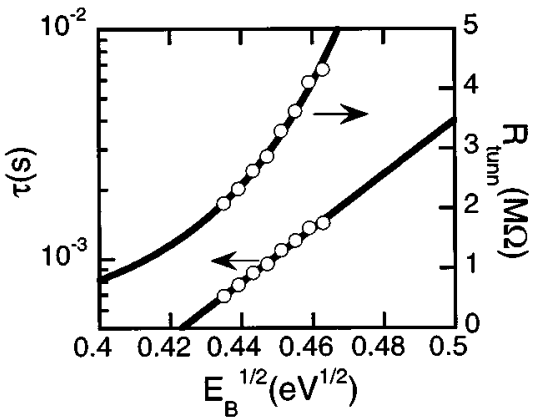

FIG. 5. Tunneling time $\tau$ and tunneling resistance $R_{\text {tunn }}$ as a function of the square root of the tunneling barrier height $E_{B}$.

this result, we observe that in order to achieve higher frequency operation one has to decrease the tunneling resistance. This can be accomplished by the changing tunneling barrier and SAQD composition and the tunneling barrier thickness. For that to be successfully implemented, attention must be paid also to the strain fields that deform the tunneling barriers underneath the substrate. The dependence of $f_{\text {peak }}$ with the inverse of the tunneling barrier thickness is not exponential as one would expect. In reality, the dependence with the thickness is more complicated since strain fields decay exponentially from the SAQD/tunneling barrier interface. The tunneling barrier height decreases considerably as well as the tunneling resistance, due to strain modulation of the conduction band.

The effect of the temperature on the charging process, and therefore the tunneling time and tunneling resistance, can be shown using the Debye model and it has been demonstrated elsewhere for a system with small metallic particles. ${ }^{15}$ One would expect that the tunneling time is inversely proportional to the temperature, and therefore at $200 \mathrm{~K}$ the tunneling times for the particular structure studied in this work would be approximately $1 \mu \mathrm{s}$.

In summary, we have investigated the loading process of electron in InAs SAQD's. This system could be described very precisely by an equivalent circuit with a Debye-type relaxation mechanism. Debye curves for several voltages demonstrated the exponential dependence of the tunneling time with the barrier height and consequently tunneling resistance. The density of states could be numerically extracted and it was consistent with TEM and AFM experiments and the picture of single electron charging for this system. The charging energy for electron in InAs SAQD's was larger than size fluctuation effects on the quantum confinement of this system, demonstrating an attractive path for the application of this system in single electron devices.

The authors would like to acknowledge financial support from NSF and QUEST. G.M.R. would like to acknowledge financial support from the Brazilian agency CNPq. J.M.G. acknowledges financial support from the Spanish Ministry of Education and Science.
*Present address: Hewlett-Packard Labs, 3500 Deer Creek Road, Building 26U, MS 26U12 Palo Alto, California 94304. Electronic address: medeiros@hpl.hp.com

${ }^{1}$ P. M. Petroff and G. Medeiros-Ribeiro, MRS Bull. 21, 50 (1996).
${ }^{2}$ R. Mirin, A. Gossard, and J. Bowers, Electron. Lett. 32, 1732 (1996).

${ }^{3}$ N. Kirstaedter, O. G. Schmidt, N. N. Ledentsov, D. Bimberg, V. M. Ustinov, A. Yu. Egorov, A. E. Zhukov, M. V. Maximov, P. 
S. Kop'ev, and Zh. I. Alferov, Appl. Phys. Lett. 69, 1226 (1996).

${ }^{4}$ K. Imamura, Y. Sugiyama, Y. Nakata, S. Muto, and N. Yokoyama, Jpn. J. Appl. Phys., Part 2 34, L1445 (1995).

${ }^{5}$ G. Medeiros-Ribeiro, D. Leonard, and P. M. Petroff, Appl. Phys. Lett. 66, 1767 (1995).

${ }^{6}$ H. Drexler, D. Leonard, W. Hansen, J. P. Kotthaus, and P. M. Petroff, Phys. Rev. Lett. 73, 2252 (1994).

${ }^{7}$ M. Fricke, A. Lorke, J. P. Kotthaus, G. Medeiros-Ribeiro, and P. M. Petroff, Europhys. Lett. 36, 197 (1996).

${ }^{8}$ S. Anand, N. Carlsson, M. -E. Pistol, L. Samuelson, and W. Seifert, Appl. Phys. Lett. 67, 3016 (1995).

${ }^{9}$ G. Medeiros-Ribeiro, F. G. Pikus, P. M. Petroff, and A. L. Efros,
Phys. Rev. B 55, 1568 (1997).

${ }^{10}$ J. P. Kotthaus (private communication).

${ }^{11}$ M. E. Rubin, G. Medeiros-Ribeiro, J. J. O’Shea, M. A. Chin, E. Y. Lee, P. M. Petroff, and V. Narayanamurti, Phys. Rev. Lett. 77, 5268 (1996).

${ }^{12}$ R. C. Ashoori, Ph.D. dissertation, Cornell University, 1991.

${ }^{13}$ E. H. Nicollian and A. Goetzberger, Bell Syst. Tech. J. 46, 1055 (1967).

${ }^{14}$ J. Lambe and R. C. Jaklevic, Phys. Rev. Lett. 22, 1371 (1969).

${ }^{15}$ R. E. Cavicchi and R. H. Silsbee, Phys. Rev. B 37, 706 (1988).

${ }^{16} \mathrm{G}$. Medeiros-Ribeiro, Ph.D. dissertation, Universidade Federal de Minas Gerais, 1996. 